\title{
DETECTING IRRIGATION EVENTS USING SENTINEL-1 DATA
}

\author{
Hassan Bazzi ${ }^{1}$, Nicolas Baghdadi ${ }^{1}$, Ibrahim Fayad ${ }^{1}$, Mehrez Zribi ${ }^{2}$, Valerie Demarez ${ }^{2}$, Yann Pageot ${ }^{2}$ \\ and Hatem Belhouchette ${ }^{3}$
}

\author{
${ }^{1}$ INRAE, UMR TETIS, University of Montpellier, 34093 Montpellier Cedex 5, France \\ ${ }^{2}$ CESBIO, CNRS, 31401 Toulouse cedex 9, France \\ ${ }^{3}$ CIHEAM-IAMM, UMR-System, 34090 Montpellier, France; belhouchette@iamm.fr
}

\begin{abstract}
Better management of water consumption in irrigated agriculture is essential in order to save water resources. The objective of this study is to propose a new model capable of detecting the irrigation events using the Sentinel-1 (S1) Cband SAR (synthetic-aperture radar) in a near real-time approach. The proposed irrigation detection model relies on the change detection in the $\mathrm{S} 1$ backscattering coefficients at plot scale. A tree-based approach has been constructed to detect irrigation events by studying the behavior of the S1 backscattering coefficients following irrigation events at plot scale over three study sites located in Montpellier (southeast France), Tarbes (southwest France) and Catalonia (northeast Spain). Auxiliary data such as the NDVI (Normalized Difference Vegetation Index) and the soil moisture estimations were integrated as additional filters to reduce ambiguities related to vegetation growth and surface roughness. The results shows that the proposed model was capable of detecting $84 \%$ of the irrigation events over Montpellier. Over Catalonia site, $90.2 \%$ of the non-irrigated plots had no detected irrigation events whereas $72.4 \%$ of the irrigated plots had one and more detected irrigation events. In Tarbes, the analysis shows that irrigation events could still be detected even in the presence of abundant rainfall events during the summer season.
\end{abstract}

Index Terms - Irrigation Events, Sentinel-1, Change Detection, Near real-time

\section{INTRODUCTION}

A spatial quantification of the irrigation extent and timing is of great importance to support the management of irrigated agricultural policies [1]. Recently, huge effort has been performed to quantify the extent and spatial distribution of irrigated areas using remote sensing optical and radar data[2]-[4]. Optical images including mainly MODIS (Moderate Resolution Imaging Spectroradiometer) and Landsat [5] has been widely used to map irrigated areas. Superior efficiency of the irrigation extent mapping has been proven using the synthetic aperture radar (SAR) data [2]. The key point for using SAR data to map irrigated areas is that the backscattered SAR signal is sensitive to the soil water content, which obviously increases after an irrigation event. Although huge effort has been already performed to quantify the spatial extent of irrigated areas at plot or basin scales, the timing and frequency of irrigation has not yet received important attention. Despite the great importance of detecting irrigation events using satellite data to monitor the water consumption, only few studies have reported methodologies capable of detecting the irrigation events at plot scale. Among few studies dealing with irrigation event detection, Chen et al [6] used MODIS and Landsat images to detect irrigation timing and frequency where they achieved an overall accuracy of $87 \%$ in detecting the irrigation events. However, the proposed method remains regional and crop type dependent.

To detect irrigation events, SAR data could provide valuable information about the irrigation episodes since the SAR backscattering signal is sensitive to the soil water content [7] . With the increase of the soil moisture values following an irrigation event, SAR data could be powerful for detecting irrigation events. However, the detection of irrigation events using SAR data could be constrained by several factors including the satellite characteristics (wavelength, revisit time) and/or vegetation characteristics (crop type, vegetation density...).

In the context of detecting irrigation events at plot scale using SAR data, a near real-time irrigation detection model using Sentinel-1 time series is proposed in this study. A tree-like approach for detecting irrigation events was developed based on the change detection of the SAR signal at plot scale cojointly with the change detection of SAR signal obtained at grid scale $(10 \mathrm{~km} \times 10 \mathrm{~km})$. Grid scale SAR signal has been used as a representative of existence or absence of rainfall events. In order to reduce the effect of the vegetation cover and the surface roughness several filters were introduced in the proposed tree-based approach including NDVI values and soil moisture estimations.

\section{MATERIALS AND METHODS}

\subsection{Datasets}


In this study, three different sites were examined over Montpellier, Catalonia (two semi-arid areas) and Tarbes (humid) (Figure 1). In the three regions, irrigation mainly occurs in the summer season between May and October of each year. Three irrigated plots over Montpellier were examined with registered irrigation dates for the period between May and October 2017 with 33 irrigation episodes. Over Catalonia, the General Direction of Rural Development of the Generalist provides the SIGPAC (Geographic Information System for agricultural parcels) data for agricultural plots. Each agricultural plot is registered for being either irrigated or non-irrigated. Finally, from a field campaign conducted in 2017, 150 irrigated maize plots were localized over the Adour Amont basin near Tarbes city.

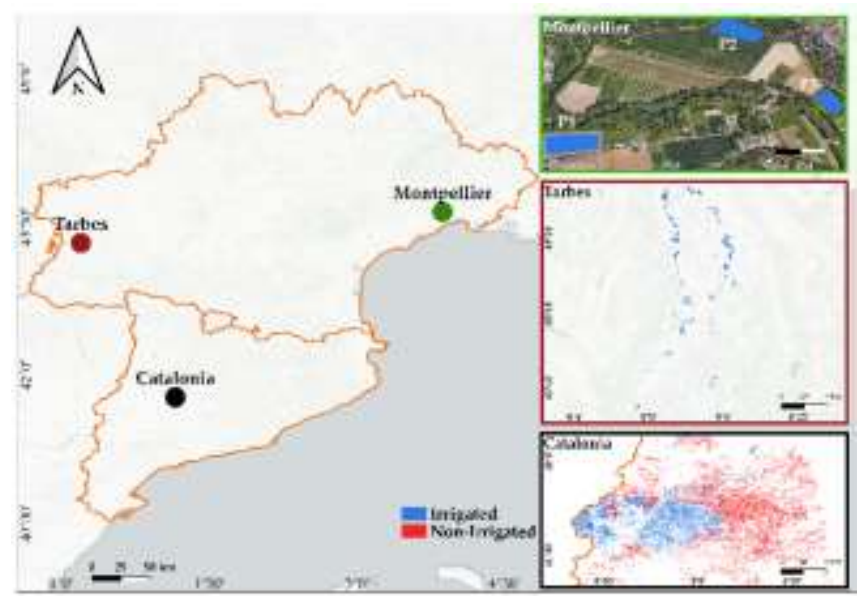

Figure 1. Location of the three studied sites in Montpellier Catalonia and Tarbes. Irrigated and non-irrigated plots are presented in blue and red respectively.

\subsection{SAR Backscattering at Plot and Grid Scale}

A total of 348 C-band $(5.405 \mathrm{GHz}) \mathrm{S} 1 \mathrm{SAR}$ images over the 3 study sites acquired by S1A and S1B in both ascending (afternoon at 18h00 UT) and descending modes (morning at 06h00 UT) were downloaded and calibrated using the S1 toolbox developed by ESA (European Space Agency). For the three study sites, the average S1 SAR backscattering coefficients $\left(\sigma^{0}\right)$ in VV polarization and the incidence angle values $(\theta)$, were calculated at both plot scale $\left(\sigma_{P}^{0}\right)$ and grid scale $\left(\sigma_{G}^{0}\right)(10 \mathrm{~km} \mathrm{x} 10 \mathrm{~km})$.

The proposed method is based on detecting the change in the $\sigma_{P}^{0}$ backscattering coefficient at plot scale. When the surface soil moisture (SSM) increases between two consecutive SAR acquisitions, $\sigma_{P}^{0}$-value between these two dates increases.

Rainfall events and irrigation events are both considered as a water supplement and thus may have the same effect on the value of $\Delta \sigma_{P}^{0}$. In this study, information about rainfall has been determined through the $\sigma^{\circ}$ value obtained at grid scale $(10 \mathrm{~km} \times 10 \mathrm{~km})$. We assume here that if the bare soil plots with low vegetation cover within the spatial extent of $10 \mathrm{~km}$ x $10 \mathrm{~km}$ show an increase of the SAR backscattering signal (increase in SSM values) between two consecutive radar acquisitions we can say that a rainfall event occurred.

\subsection{Auxiliary filters}

To minimize the effect of vegetation growth on the radar signal, we propose to smooth the temporal series of the SAR signal which gives the general behavior of the vegetation contribution in the SAR signal [8]. At each SAR date, a smoothing value $\sigma_{\text {smooth }}^{0}$ is obtained by applying a Gaussian smooth on all dates before this date (from $t_{0}$ to $t_{i}$ ). We obtain the index $S=\sigma_{P_{t i}}^{0}-\sigma_{\text {smooth }}^{0}$. If the value of $S<0$ then the point should not further considered for irrigation detection.

Surface soil moisture values at plot scale $S S M_{P}$ were used to help confirm whether a point is an irrigation point or not in the case of slight change in $\Delta \sigma_{P}^{0}$. The stability or slight decrease of the SAR signal at plot scale between $t_{i}$ and $t_{i-1}$ could be interpreted as an irrigation event if and only if the $\sigma^{0}$ at time $t_{i-1}$ already attains high values (due to irrigation or rainfall). To insure this situation, we say that at time $t_{i-1}$, SSM estimation should be greater than or equals to 20 vol. $\%$.

During the sowing or harvesting periods, the surface roughness increases due to soil work which causes an increase in the $\sigma_{P}^{0}$. For each detected irrigation event, we obtain the difference between the NDVI value at the detected irrigation event and the next NDVI value of the next optical image after 20 to 30 days $\left(\Delta N D V I=N D V I_{t i}-\right.$ $\left.N D V I_{\text {ti+(20 to } 30 \text { days })}\right)$.

NDVI values were obtained from Sentinel-2 images downloaded over the three study sites for the same period of $\mathrm{S} 1$ acquisition. If the $N D V I_{t i}$ value is less than 0.4 (bare soil conditions with small vegetation cover) and $\triangle N D V I \leq$ 0.1 then the point is a falsely detected irrigation point, and is eliminated.

\subsection{Decision Tree Irrigation Detection Model:}

A value of $\Delta \sigma_{P}^{0}$ value less than $-0.5 \mathrm{~dB}$ is considered as nonirrigation point. Otherwise, the smoothed vegetation descriptor $S$ is checked and the point is considered as nonirrigation if $S<0$. If $S$ is positive, the point is considered as non-irrigation if the $S S M_{P}<15 \mathrm{vol} . \%$ with $N D V I_{t i} \leq 0.5$ When the studied point arrives to pass by all the previous filters, the chance of having a water supplement (irrigation or rainfall) increases and the change of SAR signal at grid scale is required in order to eliminate the irrigation-rainfall ambiguity. Filters applied at grid scale could be divided into four main cases:

- Case i: If $\Delta \sigma_{G}^{0} \geq 1 d B$ then a rainfall event have occurred and the point is not an irrigation point.

- Case ii: If $S S M_{G}>20 \mathrm{vol} . \%$ then a rainfall event probably occurred before and there is low chance to have an irrigation event (humid soil conditions at basin scale). 
- Case iii: If $0.5 \leq \Delta \sigma_{G}^{0} \leq 1 \mathrm{~dB}$ we check the value of $\Delta \sigma_{P}^{0}$ for two cases:

* Case iii.1: If $\Delta \sigma_{P}^{0} \leq 0.5$ then no irrigation took place

- Case iii.2: If $\Delta \sigma_{P}^{0}>0.5$ and $\Delta\left(\Delta \sigma_{P}^{0}-\Delta \sigma_{G}^{0}\right) \geq 1$ then it is considered as irrigation point with high probability

- Case iv: If $\Delta \sigma_{G}^{0} \leq 0.5 d B$ then we check the $\Delta \sigma_{P}^{0}$ for four different cases:

* Case iv.1: $\Delta \sigma_{P}^{0} \geq 1 d B$ then the point is an irrigation point with high probability

* Case iv.2: $0.5 \leq \Delta \sigma_{P}^{0}<1 d B$ then the point is an irrigation point with medium probability if and only if $S S M_{P} \geq 20$ vol. \% OR $\Delta \geq 1.5 \mathrm{~dB}$

* Case iv.3: $0 \leq \Delta \sigma_{P}^{0}<0.5 d B$ then the point is an irrigation point with low probability if and only if $S_{S S M} \geq 20$ vol. $\%$ OR $\Delta \geq 2 d B$

* Case iv.4: $-0.5 \leq \Delta \sigma_{P}^{0}<0 d B$ then the point is an irrigation point with low probablity if and only if $S S M_{P} \geq 20$ vol. $\%$ AND the previous point at $t_{i-1}$ is a high probability irrigation point or a rainfall point $\left(\Delta \sigma_{G}^{0} \geq 1 d B\right)$

\section{RESULTS}

Table1 shows the results obtained on the three plots of Montpellier site. Among the 33 irrigation events, 28 irrigation episodes have been detected with a success rate of $84.8 \%$. However, a total error of five falsely detected irrigation events has been registered over the three plots. The falsely detected irrigation points was found to be corresponding to the saturation of the radar signal caused by very well developed vegetation cover in plots P1 (maize) and P3 (Sorghum).

Table 1. Irrigation events detected over three plots in Montpellier, South-east France.

\begin{tabular}{cccc}
\hline Plot & $\begin{array}{c}\text { Irrigation } \\
\text { events }\end{array}$ & $\begin{array}{c}\text { Detected } \\
\text { events }\end{array}$ & $\begin{array}{c}\text { False } \\
\text { detection }\end{array}$ \\
\hline P1 & 15 & 12 & 2 \\
P2 & 13 & 11 & 1 \\
P3 & 5 & 5 & 2 \\
Total & 33 & 28 & 5 \\
\hline
\end{tabular}

Over Catalonia, the model was applied over both irrigated and non-irrigated plots $(\sim 150,000$ plots $)$ to analyze the effectiveness of true and false irrigation detections. Figure 2 presents the temporal profile of $\sigma_{\mathrm{P}}^{0}$ and $\sigma_{\mathrm{G}}^{0}$ over the period between April 2018 and November 2018 (evening acquisitions) along with the irrigation episodes detected for an irrigated maize plot. Eleven irrigation episodes have been detected on the maize plots. The irrigation episodes correspond to the dry summer period (between 25 April and 30 September).

The histogram in Figure 3 represents the number of events detected over irrigated and non-irrigated plots in Catalonia using the morning acquisition. The distribution shows that



Figure 2. Irrigation detection over agricultural plots in Catalonia, Spain for irrigated maize plot

$68.3 \%$ of the non-irrigated plots had no detected irrigation events for the period between September 2017 and December 2018 (82 SAR images). Moreover, $20.3 \%$ of the nonirrigated plots encounter only one detected event. Therefore, $88.6 \%$ of the non-irrigated plots have maximum one detected irrigation point in the morning acquisition mode. On the other hand, only $12.1 \%$ of the irrigated plots failed to register any irrigation event. Thus, $87.9 \%$ of the irrigated plots have one and more detected irrigation points. The percentage then increases gradually where $54.9 \%$ of the irrigated plots have between two and five detected irrigation points. In addition, $20.4 \%$ of the irrigated plots encounter between five and ten detected irrigation points.

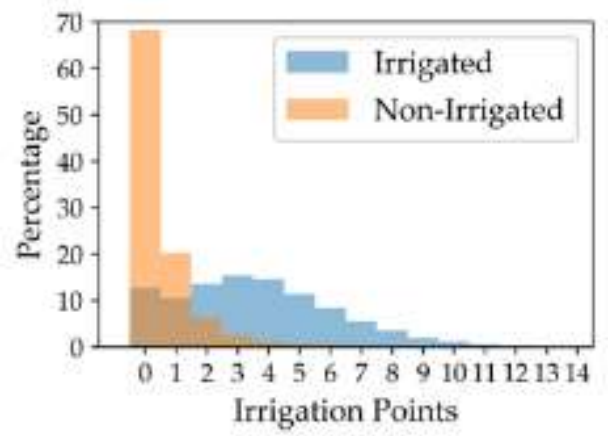

Figure 3. Distribution of the irrigation points detected over irrigated (blue) and non-irrigated (orange) plots in Catalonia. Figure 4 represents the distribution of the number of irrigation episodes detected over irrigated plots in Catalonia, Spain as a function of months. The results show that most of the detected irrigation episodes exist within the period 
between March 2018 and September 2018. Indeed, 80.0\% of the total detected episodes exist within the period between 01 March 2018 and 30 September 2018.

Figure 5 presents the histogram of the distribution of the number of events detected over irrigated maize plots in Tarbes for morning acquisition. The distribution shows that shows that using the morning acquisition, $91 \%$ of the irrigated plots has one or more detected irrigation events and $75 \%$ of the plots have two and more detected irrigation events. The low number of detected irrigation events in Tarbes, compared to those obtained over Catalonia was expected since Tarbes is a humid region and the frequency of irrigation is less than that in the arid or semi-arid regions.

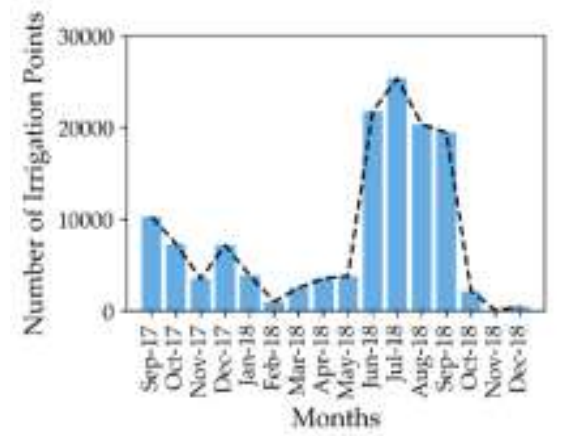

Figure 4. Distribution of the irrigation points detected over irrigated plots in Catalonia Spain as a function of months

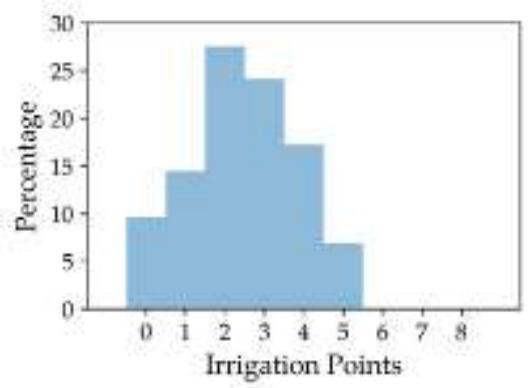

Figure 5. Distribution of the irrigation points detected over irrigated plots in Tarbes, France for (a) morning SAR acquisition

\section{CONCLUSIONS}

This paper proposes a new approach for detecting irrigation events at plot scale in a near real-time scenario. The proposed method is a decision tree-based approach for detecting irrigation events using the change detection in the S1 SAR backscattering coefficients at plot scale. Several filters were applied in order to remove the ambiguity between irrigation events and rainfall, vegetation development and soil surface roughness. To ensure the transferability and the possible operational application of the proposed approach, three study sites were examined (Montpellier, Catalonia and Tarbes) with two different climatic properties. Our irrigation events detection method opens the way toward building new semi-supervised approaches for irrigated area mapping at plot scale. This method could be used to create a dataset of irrigated/non-irrigated labelled samples in order to be used in a machine-learning model that could be more efficient.

\section{ACKNOWLEDGMENT}

The authors would like to thank the National Center for Space Studies (CNES, TOSCA project), the National Institute for Agronomic and Environmental Research (INRAE) and the Mediterranean Agronomic Institute of Montpellier (CIHEAM-IAMM).

\section{REFERENCES}

[1] M. Ozdogan, Y. Yang, G. Allez, et C. Cervantes, "Remote Sensing of Irrigated Agriculture: Opportunities and Challenges », Remote Sensing, vol. 2, $\mathrm{n}^{\mathrm{o}}$ 9, p. 2274-2304, sept. 2010, doi: 10.3390/rs2092274.

[2] H. Bazzi et al., «Mapping Irrigated Areas Using Sentinel-1 Time Series in Catalonia, Spain », Remote Sensing, vol. 11, n ${ }^{\circ} 15$, p. 1836, août 2019, doi: 10.3390/rs11151836.

[3] Q. Gao, M. Zribi, M. Escorihuela, N. Baghdadi, et P. Segui, «Irrigation Mapping Using Sentinel-1 Time Series at Field Scale », Remote Sensing, vol. 10, no 9, p. 1495 , sept. 2018, doi: 10.3390/rs10091495.

[4] H. Bazzi, D. Ienco, N. Baghdadi, M. Zribi, et V. Demarez, «Distilling Before Refine: Spatio-Temporal Transfer Learning for Mapping Irrigated Areas Using Sentinel-1 Time Series », IEEE Geosci. Remote Sensing Lett., p. 1-5, 2020, doi: 10.1109/LGRS.2019.2960625.

[5] Y. Chen et al., « Detecting irrigation extent, frequency, and timing in a heterogeneous arid agricultural region using MODIS time series, Landsat imagery, and ancillary data », Remote Sensing of Environment, vol. 204, p. 197-211, 2018.

[6] Y. Chen et al., « Detecting irrigation extent, frequency, and timing in a heterogeneous arid agricultural region using MODIS time series, Landsat imagery, and ancillary data », Remote Sensing of Environment, vol. 204, p. 197-211, 2018.

[7] N. Baghdadi et al., « Estimating surface soil moisture from TerraSAR-X data over two small catchments in the Sahelian Part of Western Niger », Remote Sensing, vol. 3, n ${ }^{\circ}$ 6, p. 1266-1283, 2011.

[8] A. Nasrallah et al., « Sentinel-1 Data for Winter Wheat Phenology Monitoring and Mapping », Remote Sensing, vol. $11, \mathrm{n}^{\circ} 19$, p. 2228 , sept. 2019, doi: 10.3390/rs11192228. 